\title{
LEARNING ENGAGEMENT, LEARNING OUTCOMES AND LEARNING GAINS: LESSONS FROM LA
}

\author{
Dirk Tempelaar ${ }^{1}$, Bart Rienties ${ }^{2}$ and Quan Nguyen ${ }^{2}$ \\ ${ }^{I}$ Maastricht University School of Business and Economics, Tongersestraat 53, 6211 LM, Maastricht, Netherlands \\ ${ }^{2}$ Open University UK, Institute of Educational Technology, Walton Hal, Milton Keynes, MK7 6AA, UK
}

\begin{abstract}
Learning analytic models are built upon traces students leave in technology-enhanced learning platforms as the digital footprints of their learning processes. Learning analytics uses these traces of learning engagement to predict performance and provide learning feedback to students and teachers when these predictions signal the risk of failing a course, or even dropping-out. But not all of these trace variables act as stable and reliable predictors of course performance. In previous research, the authors concluded that trace variables of product type, such as mastery, do a better job than trace variables of process type, such as clicks or time-on-task, in predicting performance. In this study, we extend this analysis by focusing on learning gains rather than learning outcomes as the most important performance dimension. Distinguishing two different levels of initial proficiency, our empirical analysis into the learning of mathematics by first-year university students indicates that the lack of stability of the engagement types of process type is mainly explained by learning pattern found in students of high initial proficiency. For these students, high levels of engagement lead to lower, rather than higher, predicted learning outcomes. Amongst students with lower initial proficiency, higher levels of engagement play a different role.
\end{abstract}

\section{KEYWORDS}

Blended Learning, e-Tutorials, Learning Analytics, Learning Engagement, Learning Traces, Process and Product Data

\section{INTRODUCTION}

Learning Analytics (LA) has the prime aim to improve the study success of students by collecting data about the way students learn, designing models that can extrapolate observed learning behaviours into predictions of learning outcomes, and provide learning feedback to students and teachers in case those predictions signal a risk of unsuccessful learning, or even drop-out (Ifenthaler, 2015; Ifenthaler, Mah, \& Yau, 2019). An important source of data applied in predictive modelling is trace data generated by technology-enhanced learning environments that represent the digital footprints of the learning of students in these learning tools. LA is not without challenges (Ifenthaler et al., 2019): how to define study success, and what trace variables to choose to provide reliable predictions of that study success, are amongst such challenges.

Early applications of LA focussed strongly on signalling the risk of drop-out or more generally: failing the course. Failing is determined by course performance measures as expressed in terms of grades. Best predictors of performance on final exams that are timely enough to allow for educational interventions are typically early, of course, performance measures, such as scores on an intermediate test or quizzes. All of these are outcome measures that characterise the level of proficiency of the students, but not necessarily the amount of learning taking place in the course. And that is actually what course learning objectives are about: to learn new concepts, to increase rather than establish students' levels of proficiency.

This growth objective is best represented by the concept of learning gain (Rogaten and Rienties, 2018; Rogaten et al., 2018). In this learning gain literature, a rich set of learning gains is distinguished, ranging from learning gains of affective type, through behavioural types, to cognitive types. The difficulty in applying these concepts is in the measurement. That is especially true for learning gains of affective and behavioural types, but even if one limits the focus on learning gains of cognitive type only, as we will do in this study, measurement is a challenge. Some empirical studies use indirect measures such as the use of self-report surveys by students asking to quantify how much they learned, while other studies applied 
objective tests in a pre- and post-test design (Rogaten and Rienties, 2018; Rogaten et al., 2018). But where the use of objective testing is generally to be preferred above self-report surveys, the measurement of cognitive learning gains would require the use of equivalent pre- and post-tests, as in progress testing, which is hardly ever done.

The aim of the current study is to investigate potential differences in typical LA type of models with learning outcomes versus learning gains as response variables and trace variables of student engagement as predictor variables. Research questions in this context are: how do these prediction models differ between the two different response variables? And as a follow-up question: what role do the different trace variables, all representing different facets of student engagement, in these prediction models? This last research question connects to previous research of the authors (Tempelaar et al., 2015, 2017, 2018a), where we found that some measures of engagement, such as time-on-task or the intensity of using worked-out examples, are negatively related to course outcomes in multivariate prediction equations, rather than the positive relationship hypothesized. Does that outcome hold when we predict learning gain rather than learning outcome, is the research question of this contribution, thereby providing an immediate extension of our Tempelaar (2017) study?

\section{METHODS}

\subsection{Context of the Empirical Study}

This study takes place in a large-scale introductory mathematics and statistics course for first-year undergraduate students in a business and economics program in the Netherlands. The educational system is best described as 'blended' or 'hybrid' applying the flipped classroom approach. The main component is face-to-face: Problem-Based Learning (PBL), in small groups (14 students), coached by content expert tutors (in 78 parallel tutorial groups). Participation in tutorial groups is required. Optional is the online component of the blend: the use of the two e-tutorials SOWISO (https://sowiso.nl/) and MyStatLab (MSL) (Nguyen et al., 2016; Tempelaar, 2017; Tempelaar et al., 2015; 2017). This design is based on the philosophy of student-centred education, placing the responsibility for making educational choices primarily on the student. Since most of the learning takes place during self-study outside class through the e-tutorials or other learning materials, class time is used to discuss solving advanced problems. Thus, the instructional format shares most characteristics of the flipped-classroom design. Using and achieving good scores in the e-tutorial practice modes is incentivized by providing bonus points for good performance in quizzes that are taken every two weeks and consist of items that are drawn from the same item pools applied in the practising mode. This approach was chosen to encourage students with limited prior knowledge to make intensive use of the e-tutorials.

The subject of this study is the full 2018/2019 cohort of students (1208 students in total, 1055 students included in this study who have full data records). A large diversity of the student population was present: only $20 \%$ were educated in the Dutch high school system, against $80 \%$ being international students, with 56 nationalities present. A large share of students was of European nationality, with only $3.9 \%$ of students from outside Europe. High school systems in Europe differ strongly, most particularly in the teaching of mathematics and statistics. Therefore, it is crucial that this introductory module is flexible and allows for individual learning paths. Students spend on average 28.3 hours in SOWISO and 25.0 hours in MSL, which is $30 \%$ to $35 \%$ of the available time of 80 hours for learning both topics. This study focuses on learning engagement of students in only one of the two e-tutorials, to know the math related SOWISO tool, for several reasons: the availability of trace data for several types of engagement (see next section), reliable time-on-task data by correction for inactive time, and the availability of prior education data regarding mathematics, but not statistics. 


\subsection{Instruments and Procedure}

Both e-tutorial systems SOWISO and MSL follow test-directed learning and practising approach. Each step in the learning process is initiated by a question, and students are encouraged to (attempt to) answer each question. If a student does not master a question (completely), she/he can either ask for hints to solve the problem step-by-step, or ask for a fully worked example. After receiving feedback, a new version of the problem loads (parameter based) to allow the student to demonstrate his/her newly acquired mastery. Students' revealed preferences for learning strategies are related to their learning dispositions, as we demonstrated in previous research (see Nguyen et al. (2016) and Tempelaar et al. (2017, $2018 \mathrm{a}, \mathrm{b}$ ) for the use of worked-examples in SOWISO, and (Tempelaar, 2017) for the use of worked-examples in MSL). This study extends Nguyen et al. (2016) and Tempelaar et al. $(2018 \mathrm{a}, \mathrm{b})$ by investigating three learning strategies in the SOWISO tool: worked examples, and supported and tutored problem-solving.

Figure 1 demonstrates the implementation of the alternative learning strategies students can opt for a sample exercise:

- Check: the untutored problem-solving approach, offering only correctness feedback after problem-solving;

- Hint: the tutored problem-solving approach, offering feedback and hints to assist the student in the several problem-solving steps;

- Solution: the worked-examples approach;

- Theory: asking for a short explanation of the mathematical principle.

Week6: Functions of two variables: Basic notions

Visualizing bivariate functions (exercise id: 1549)

Consider the function

$$
f(x, y)=\mathrm{e}^{-x^{2}-y^{2}}
$$

Its graph is displayed in the figure below.

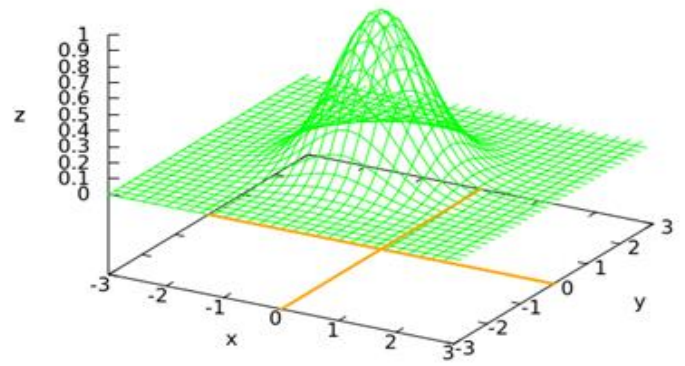

At a particular point $[a, b]$ the level curve going through that point is the unit circle. What is the value $f(a, b)$ ?

$f(a, b)=$

$\checkmark$ Check

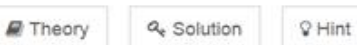

Figure 1. Sample of SOWISO exercise with the options Check, Theory, Solution, and Hint

Our study combines trace data of the SOWISO e-tutorial with prior-education SIS data and course performance data. Clicks in the two e-tutorial systems represent an important part of that trace data, but trace data can include more than click data only. Azevedo (Azevedo et al., 2013) distinguishes between trace data of product and process type, where click data is part of the category of process data. In this study, we will combine both process data, as, e.g. the clicks to initiate the learning supports of Check, Hint, Solution and Theory mentioned above, but also product data, as, e.g. mastery in the tool, as discussed below. SOWISO reporting options of trace data are very broad, requiring making selections from the data. First, all dynamic trace data were aggregated over time, to arrive at static, full course period accounts of trace data. Second, 
from the large array of trace variables, a selection was made by focusing on process variables most strongly connected to alternative learning strategies.

In total, four trace variables were selected:

- Mastery in the tool, the proportion of exercises successfully solved as product indicator;

- Attempts: the total number of attempts of individual exercises;

- Hints: the total number of Hints called.

- Solutions: the total number of worked-out examples called.

The Attempts variable is cumulative: it is the sum of calls for hints, for worked-out examples, and untutored problem-solving attempts. The system does not trace the number of clicks for the Check option, but that number follows from removing the Solution and Hints activities from the total number of Attempts.

Pass/fail decisions for the course are based on four course outcome measures: performance in the exam, both for mathematics (MathExam) and statistics (StatsExam), and the aggregated performance in the three quizzes for both topics: MathQuiz and StatsQuiz. The exam brings 40 credits, against 6 credits for quizzes, indicating the limited weight of the quizzes (13\%). Because of missing adequate trace and prior education data for the statistics part, predictive modelling will be limited to the two mathematical performance variables, indicated as Exam and Quiz.

Prior education measures are based on self-reported accounts of the secondary mathematics program at the secondary level. Most countries distinguish three levels of math teaching in upper-level high school: the highest level, preparing for science university studies, the intermediate level, preparing for social sciences university studies, and the lower level, preparing for humanities. Examples are the levels HI, SL, and SL studies of the International Baccalaureate (IB) program. To be admissible to the school, at least the intermediate level is required. That allows describing the level of prior education by an indicator variable, labelled as MathMajor, having a value of 1 for students educated in the highest track, and 0 for students educated in the intermediate track. Shares of these two tracks are $37.1 \%$ and $62.9 \%$, respectively.

\subsection{Learning Gains and Learning Outcomes}

Although learning gain, how much students actually learn by taking a course, is obviously the more important aim than the learning outcome, how much students know at the end of the course by combining newly learned knowledge with prior knowledge, most empirical studies in learning analytics focus on learning outcomes. This is best explained by the simple reason that student assessment in any course brings exact measures of learning outcomes in the form of exam and quiz grades, whereas learning gain is defined by the growth in knowledge throughout the course, and thus requires the measurement of the starting level. Such measures are however difficult to operationalize, and for that reason, many empirical studies in learning gains take the step to equate learning gains with learning outcomes. In our context, we collected two measures that may qualify as a proxy of the level cognitive understanding at the start of the course: the outcome of a diagnostic entry test, and self-reported data on prior mathematics education at the secondary level. In a preliminary analysis, we investigated the potential of the diagnostic test score as a measure of starting level of proficiency and concluded that it was less fit to play that role. There are two different aspects to this misfit. First, the diagnostic test is used as an instrument to inform students how well they are prepared to take the course. So there is an issue with coverage: the entry test covers topics at a foundational level, rather than topics taught in the course itself. Second: students administer the diagnostic test outside class, which gives rise to very different response patterns. Some students do not take the test seriously, especially when they know they are well prepared, given their high level of proficiency. Other students, especially at the other end of the continuum of preparedness, tend to take the entry test 'too' seriously and achieve scores that are higher than their ready knowledge. The prior education measure does not share these issues. It is less fine-grained in that it distinguishes only two different levels, but the coverage of the advanced level program overlaps to large extent topics taught in our course, and there are no issues with the administration. Therefore, we opted for the prior education dummy variable to describe two different proficiency levels at the start of the course: one for students of the advanced track, one for students of the intermediate track.

In terms of model design, it is not the initial level of proficiency of the two groups itself that is crucial in the learning gain approach, but the difference between these two levels. We estimate that difference using multivariate regression models, where learning outcome is the response variable, and trace variables together with the prior education dummy variable serve the role of predictor variables. The estimate of the regression 
coefficient of the prior education dummy represents the difference in prior proficiency between the two groups expressed in units of the learning outcome variable. In that context, the learning gain equals the difference between learning outcome itself, and the estimated effect of prior education.

\section{RESULTS}

\subsection{Learning Outcomes and Traces of Learning}

Correlating course outcomes (Exam and Quiz), track of prior education (the dummy MathMajor) with traces of engagement with the e-tutorial, both of product type (Mastery) and of process type (Attempts, Solutions, Hints, Time) produces some unexpected outcomes: see Table 1. As expected, Exam and Quiz are strongly positively related, and both are moderately positively related to having followed math classes at an advanced level in high school. The unexpected outcomes refer to the correlations between Exam, the main course outcome variable, and the several trace variables of process type. Except for Time, these correlations are non-significant or even negative: Attempts, Solutions, Hints. Correlations between the other course outcome variable, Quiz, exhibit a very different pattern: these are all significantly positive.

Table 1. Bivariate correlations of course outcomes, prior education and traces of learning in the e-tutorial; full sample

\begin{tabular}{lcccccccc}
\hline Variable & 1. & 2. & 3. & 4. & 5. & 6. & 7. & 8. \\
\hline 1. Exam & 1.000 & & & & & & & \\
2. Quiz & $.558^{* * *}$ & 1.000 & & & & & & \\
3. MathMajor & $.381^{* * *}$ & $.375^{* * *}$ & 1.000 & & & & & \\
4. Mastery & $.219^{* * *}$ & $.545^{* * *}$ & $.078^{*}$ & 1.000 & & & & \\
5. Attempts & -.037 & $.356^{* * *}$ & $-.077^{*}$ & $.778^{* * *}$ & 1.000 & & \\
6. Solutions & $-.210^{* * *}$ & $.108^{* * *}$ & $-.188^{* * *}$ & $.557^{* * *}$ & $.916^{* * *}$ & 1.000 & & \\
7. Hints & -.007 & $.104^{* * *}$ & -.052 & $.217^{* * *}$ & $.166^{* * *}$ & .056 & 1.000 & \\
8. Time & $.071^{*}$ & $.262^{* * *}$ & .009 & $.423^{* * *}$ & $.360^{* * *}$ & $.207^{* * *}$ & $.202^{* * *}$ & 1.000 \\
\hline
\end{tabular}

Note: ${ }^{* * *} \mathrm{p}<.001 ;{ }^{* *} \mathrm{p}<.01 ;{ }^{*} \mathrm{p}<.05$.

Correlations between the several engagement variables of trace type are all positive or non-significant. Number of Attempts and number of Solutions are so strongly related, that they constitute a collinear set. On average, students do 745 Attempts, of which 325 are calls for worked examples: the Solutions. This ratio of $44 \%$ of the Attempts being calls for Solutions is rather constant over students, causing a high correlation. This affects the regression in the next step of the analysis: including both Attempts and Solutions gives rise to high variance inflation factors. All regressions have been run with both Attempts and Solutions as a predictor, and in all cases, the fit of the regression model with Solutions is far better than that with Attempts. For that reason, the Attempts variable is excluded from all regression models.

The Hints variable behaves differently: not only is its correlation with Attempts no more than weak, students call on average no more than 29 Hints, what is in only $4 \%$ of their Attempts.

The regression equations explaining the two course outcomes variables, Exam and Quiz, out of e-tutorial trace variables is provided in Table 2 .

Table 2. Regressions of course outcomes on e-tutorial trace data

\begin{tabular}{lccrrrr}
\hline & Exam & & \multicolumn{3}{c}{ Quiz } \\
LA trace predictor & $\mathrm{b}$ & $\mathrm{SE}(\mathrm{b})$ & \multicolumn{1}{c}{$\beta$} & $\mathrm{b}$ & $\mathrm{SE}(\mathrm{b})$ & $\beta$ \\
\hline Mastery & 6.143 & 0.405 & $0.526^{* * *}$ & 1.556 & 0.066 & $0.736^{* * *}$ \\
Solutions & -0.006 & 0.000 & $-0.496^{* * *}$ & -0.001 & 0.000 & $-0.329^{* * *}$ \\
Hints & -0.006 & 0.002 & $-0.091^{* * *}$ & -0.001 & 0.000 & $-0.052^{* *}$ \\
Time & -0.000 & 0.000 & -0.044 & -0.000 & 0.000 & -0.005 \\
$\mathrm{R}^{2}$ & 0.222 & & & 0.370 & & \\
\hline
\end{tabular}

Note: $* * * \mathrm{p}<.001 ; * * \mathrm{p}<.01 ; * \mathrm{p}<.05$. 
Trace variables explain $22 \%$ of the variation in the Exam outcome and $37 \%$ of the variation in Quiz outcome. Since quizzes are taken in the same e-tutorial tool as students practice in, and contain items similar to those students encounter in the practice mode, it is not surprising that the Quiz outcome variable is better explained by the trace variables, than the Exam outcome variable. Remarkably, all trace variables of process type have negative or zero betas. That is: taking the mastery level of the student into account, students who call more Solutions, or who call more Hints, are predicted to achieve lower course outcomes, on average.

\subsection{Learning Gains and Traces of Learning}

To operationalize learning gains, an estimate of the cognitive abilities of the students before entering the course is required. The best estimate available is the level of prior education in high school: advanced track or intermediate track. The indicator variable MathMajor serves that role: it indicates if a student took the advanced track (MathMajor=1) or the intermediate track (MathMajor=0). Including the MathMajor variable in the regression model implies that regression intercepts and slopes differ between the two groups, based on differences in correlations. These group-specific correlations are contained in Table 3, with correlations of the students of the MathMajor track above the diagonal, and those of the MathIntermediate track below the diagonal.

Table 3. Bivariate correlations of course outcomes, prior education and traces of learning in the e-tutorial; MathMajor sample above the diagonal, MathIntermediate sample below the diagonal

\begin{tabular}{|c|c|c|c|c|c|c|c|}
\hline Variable & 1. & 2. & 3. & 5. & 6. & 7. & 8. \\
\hline 1. Exam & 1.000 & $.557^{* * *}$ & $205^{* * *}$ & -.048 & $-.253^{* * *}$ & $-.118^{*}$ & -.037 \\
\hline $\begin{array}{l}\text { 2. Quiz } \\
\text { 3. MathMajor }\end{array}$ & $.456^{* * *}$ & 1.000 & $.505^{* * *}$ & $.279^{* * *}$ & -.003 & .038 & $.148^{* *}$ \\
\hline 4. Mastery & $.214^{* * *}$ & $.574^{* * *}$ & 1.000 & $.783^{* * *}$ & $.527^{* * *}$ & $.203^{* * *}$ & $.434^{* * *}$ \\
\hline 5. Attempts & .008 & $.445^{* * *}$ & $.808^{* * *}$ & 1.000 & $.890^{* * *}$ & $.162^{* * *}$ & $.355^{\text {*** }}$ \\
\hline 6. Solutions & $-.128^{* * *}$ & $.230^{* * *}$ & $.618^{* * *}$ & $.927^{* * *}$ & 1.000 & .076 & $.218^{* * *}$ \\
\hline 7. Hints & $082^{*}$ & $179^{* * *}$ & $231^{* * *}$ & $165^{* * *}$ & 037 & 1.000 & $206^{* * *}$ \\
\hline 8. Time & $.138^{* * *}$ & $.334^{* * *}$ & $428^{* * *}$ & $.380^{* * *}$ & $224^{* * *}$ & $.194^{* * *}$ & 1.000 \\
\hline
\end{tabular}

$$
\text { Note: } * * * \mathrm{p}<.001 ; * * \mathrm{p}<.01 ; * \mathrm{p}<.05
$$

Comparing the first two rows with the first two columns makes clear that large difference exists between the two groups. Correlations between e-tutorial trace data and the two course outcomes Exam and Quiz are more positive everywhere for the students of the intermediate level track than for advanced level track. These differences in correlations show up in different regression equations, as exhibited in Table 4.

Table 4. Regressions of course outcomes on prior education and e-tutorial trace data

\begin{tabular}{lcccccc}
\hline & Exam & & \multicolumn{3}{c}{ Quiz } \\
LA trace predictor & $\mathrm{b}$ & $\mathrm{SE}(\mathrm{b})$ & $\beta$ & $\mathrm{b}$ & $\mathrm{SE}(\mathrm{b})$ & $\beta$ \\
\hline MathMajor & 3.269 & 0.339 & $0.472^{* * *}$ & 0.533 & 0.054 & $0.419^{* * *}$ \\
Mastery & 5.560 & 0.385 & $0.467^{* * *}$ & 1.463 & 0.062 & $0.684^{* * *}$ \\
Solutions & -0.005 & 0.000 & $-0.388^{* * *}$ & -0.001 & 0.000 & $-0.230^{* * *}$ \\
Hints & & & & & & \\
Time & & & & & & \\
Mastery*MathMajor & & & & & & \\
Solutions*MathMajor & -0.002 & 0.001 & $-0.120^{* *}$ & 0.000 & 0.000 & $-0.126^{* * *}$ \\
Hints*MathMajor & -0.012 & 0.003 & $-0.105^{* * *}$ & & & \\
Time*MathMajor & -0.000 & 0.000 & $-0.096^{*}$ & -0.000 & 0.000 & $-0.073^{*}$ \\
$\mathrm{R}^{2}$ & 0.324 & & & 0.455 & & \\
Note: ***p $<.001 ; * * \mathrm{p}<.01 ; * \mathrm{p}<.05$. & & & & &
\end{tabular}

The regression coefficients of the MathMajor indicator variable are positive, signalling that students from the MathMajor group score on average 23\% higher in the Exam and 37\% higher in the Quiz. Other differences between the two groups are visible from the estimates of the interaction terms. The negative effects of the trace variables of process type, Solutions, Hints and Time, accounting for the Mastery interaction term and the main effects are much stronger for the MathMajor group than for the MathIntermediate group. 


\section{DISCUSSION AND CONCLUSION}

The MathMajor variable has a strong effect on the two learning outcomes, both directly, as well as indirectly through three of the four interaction terms, as visible from Table 4. The direct effect tells us that students of the advanced track score, on average 3.3 points higher in the final exam (on a range of $0 \ldots 20$ ), and 0.53 points higher in the quiz (on a range of $0 \ldots 3$ ), than students of the intermediate math track. That direct effect is intuitive: the math topics covered in our course have a strong overlap with the curriculum of the advanced track, next to the circumstance, that one can expect the proportion of talented math student to be higher in that track, than the intermediate level track. That direct effect is our proxy of the difference between learning outcome and learning gain. If we bring that direct effect to the left side of the regression equation, the resulting equation describes predicted learning gain as a function of trace variables and interaction terms.

The interesting part of the regressions in Table 4 is the role of the trace variables. The direct effect of the trace variables is statistically non-significant, except for Solutions, telling that when controlling for Mastery level and prior education, calling more Solutions predicts lower performance. That is: the student who needs more worked-out examples to reach the same mastery level as another student needing less worked-out examples, will score less in exam and quiz, on average. There is no significant overall effect for Hints and Time. However, there are significant interaction effects for Hints, Time as well as Solutions, all with negative betas. All are telling the same story: students of the advanced track who, accounting for their mastery level, need more Solutions, Hints or Time, are expected to do less well than students of the advanced track who reached the same mastery level using fewer Solutions, Hints or Time. The effects for Hints and Time are unique to the students of the advanced track: no similar effect is at work in students of the intermediate track. The interaction effect of Solutions related to students of the advanced track, adds to the general effect of Solutions that relates all students.

In our previous research (Tempelaar, 2017; Tempelaar et al., 2017, 2018 a, b), investigating learning processes in the same course but with different year classes, we invariably found that trace variables of process type derived from learning processes in the e-tutorials play a less unambiguous role in prediction models. Their bivariate relationships with course performance variables are generally positive (as in this study, except for Solutions). However, in multivariate prediction equations that include the Mastery trace variable of product type along with the trace variables of process type, these betas of the process type trace variables are generally negative (as in this study). Telling the story that the student who needs a more intensive process to reach the same mastery level as another student, is expected to do less well in the exam and quiz. Where process that is more intensive means: more Solutions, Hints and or Time.

In the current study, we can specialize these effects by focusing on learning gains rather than learning outcomes. We find different regression models for students of the two tracks of prior education. For students educated at the advanced track who learned a substantial part of the course coverage in high school, passing the course does not require large learning gains. For these students, high levels of engagement rather than low levels of engagement represent a risk factor. They require more worked-out examples; more hints and more time on task to reach a certain mastery level signals weaker understanding and predicts lower performance. Student educated at the lower, intermediate track display a different pattern. These students require a large learning gain to pass the course. Spending more time on task and using more hints to reach a certain mastery level represents, in this case, no risk factors; only the frequent use of worked examples does.

This study is based on learning processes of students in a blended learning context, with a one-sided observation of those learning processes: we measure every detail of learning activities in the digital mode, but any learning in the face-to-face mode is left unobserved. Since the balance between these two learning modes will differ from student to student, this is a clear limitation of the context of this study.

\section{REFERENCES}

Azevedo, R., Harley, J., Trevors, G., Duffy, M., Feyzi-Behnagh, R., Bouchet, F., et al. (2013). Using trace data to examine the complex roles of cognitive, metacognitive, and emotional self-regulatory processes during learning with multi-agents systems. In R. Azevedo \& V. Aleven (Eds.), International handbook of metacognition and learning technologies, pp. 427-449. Amsterdam, The Netherlands: Springer. 
Buckingham Shum, S. \& Deakin Crick, R. (2012). Learning Dispositions and Transferable Competencies: Pedagogy, Modelling and Learning Analytics. In S. Buckingham Shum, D. Gasevic, \& R. Ferguson (Eds.). Proceedings of the 2nd International Conference on Learning Analytics and Knowledge, pp. 92-101. ACM, New York, NY, USA.

Ifenthaler, D. (2015). Learning analytics. In J. M. Spector (Ed.), Encyclopedia of educational technology, Vol. 2, pp. 447-451. Thousand Oaks, CA: Sage.

Ifenthaler, D., Mah, D.-K. and Yau, J. Y.-K. (2019). Utilising Learning Analytics for Study Success: Reflections on Current Empirical Findings. In D. Ifenthaler et al. (eds.), Utilizing Learning Analytics to Support Study Success, pp. 27-36. Switzerland: Springer Nature.

Nguyen, Q., Tempelaar, D.T., Rienties, B. and Giesbers, B. (2016). What learning analytics based prediction models tell us about feedback preferences of students. In Amirault, R., \& Visser, Y., (Eds.). (2016). e-Learners and Their Data, Part 1: Conceptual, Research, and Exploratory Perspectives. Quarterly Review of Distance Education. 17(3).

Rogaten, J. and Rienties, B. (2018). Which first-year students are making most learning gains in STEM subjects?. Higher Education Pedagogies, 3(1), pp.161-172.

Rogaten, J., Rienties, B., Sharpe, R., Cross, S., Whitelock, D., Lygo-Baker, S. and Littlejohn, A. (2018). Reviewing affective, behavioural and cognitive learning gains in higher education. Assessment \& Evaluation in Higher Education, 44(3), pp.321-337.

Tempelaar, D. (2017). How dispositional Learning Analytics helps understanding the worked-example principle. In D. G. Sampson, J. M. Spector, D. Ifenthaler, \& P. Isaias (Eds.), Proceedings 14th International Conference on Cognition and Exploratory Learning in Digital Age (CELDA 2017), pp. 117-124. International Association for Development of the Information Society, IADIS Press.

Tempelaar, D., Rienties, B. and Giesbers, B. (2015). In search for the most informative data for feedback generation: Learning analytics in a data-rich context. Computers in Human Behavior, 47, pp.157-167.

Tempelaar, D., Rienties, B. and Nguyen, Q. (2017). Towards Actionable Learning Analytics Using Dispositions. IEEE Transactions on Learning Technologies, 10(1), pp.6-16.

Tempelaar, D., Rienties, B., Mittelmeier, J. and Nguyen, Q. (2018a). Student profiling in a dispositional learning analytics application using formative assessment. Computers in Human Behavior, 78, pp.408-420..

Tempelaar, D., Rienties, B. \& Nguyen, Q. (2018b). Investigating learning strategies in a dispositional learning analytics context: the case of worked examples. In Proceedings of the International Conference on Learning Analytics and Knowledge, Sydney, Australia, March 2018 (LAK'18), p. 201-205.

Williams, A., Sun, Z., Xie, K., Garcia, E., Ashby, I., Exter, M., Largent, D., Lu, P., Szafron, D., Ahmed, S., Onuczko, T., Smith, J., Tempelaar, D. T., Bitting, K. S., Olcott Marshall, A., Christensen, E., Tseng, H., \& Walsh, J. (2016). Flipping STEM. In L. Santos Green, J. Banas, R. Perkins (Eds.), The Flipped College Classroom, Conceptualized and Re-Conceptualized, Part II, pp. 149-186. Switzerland: Springer International Publishing. 